\title{
Soft Printable Electrode Coating for Neural Interfaces
}

\author{
Michael Shur, Florian Fallegger, Elvira Pirondini, Adrien Roux, Arnaud Bichat, Quentin Barraud, \\ Grégoire Courtine, and Stéphanie P. Lacour*
}

Cite This: https://dx.doi.org/10.1021/acsabm.0c00401

Read Online

ACCESS | 世 Metrics \& More | 四 Article Recommendations

ABSTRACT: The mechanical mismatch between implantable interfaces and neural tissues may be reduced by employing soft polymeric materials. Here, we report on a simple strategy to prepare and pattern a soft electrode coating of neural interfacing devices based on a screen-printable conducting hydrogel. The coating formulation, based on polyacrylamide and poly(3,4-ethylenedioxythiophene) polystyrene sulfonate, is suitable to additive manufacturing and exhibits excellent adhesion to polydimethylsiloxane, an elastomer commonly used as a substrate in soft neural interfaces. The soft conductive coating displays a tunable elastic modulus in the 10$100 \mathrm{kPa}$ range and electrochemical properties on a par with stiff conductive inks while supporting good neural cell attachment and proliferation in vitro. Next, the soft printable hydrogel is integrated within a $4 \times 4$ microelectrode array for electrocorticography with $250 \mu \mathrm{m}$-diameter contacts. Acute recording of cortical local field potentials and electrochemical characterization preimplantation and postimplantation highlight the stability of the soft organic conductor. The overall robustness of the soft coating and its patterning method provide a promising route for a range of implantable bioelectronic applications.

KEYWORDS: conductive hydrogel, soft electrode coating, neural interfaces, electrocorticography array, microelectrodes

\section{INTRODUCTION}

A common strategy to communicate with the nervous system relies on electrical coupling of an electrode with the neural tissue. Ionic and/or electronic charge transfer across the electrode-tissue interface captures or elicits neural activity. ${ }^{1-3}$ Electrodes and their associated materials therefore ensure efficient and reliable performance for a period determined by the function and use of the specific neural interface. Electrodes are usually metallic films decorated with an engineered coating that further optimizes their physical, electrical, biological, and/ or chemical properties to ensure long-term performance. Different coating materials have been reported over the years, spanning from stiff and brittle metals and ceramics to softer and more compliant organic and nanostructured formulations. ${ }^{4-7}$ The coatings are patterned through a variety of techniques that include, but are not limited to, microfabrication methods such as evaporation and sputtering, electrodeposition, casting, and various types of printing. $6,8-11$ Recently, the call for soft and compliant neural interfaces led to the development of electrically conducting hydrogels (ECHs). ${ }^{12-14}$ The latter leverage the properties of hydrogels as soft, biomimetic, and stable three-dimensional (3D) constructs while introducing electrical conductivity within their very structure. Conducting polymers such as poly $(3,4-$ ethylenedioxy thiophene) polystyrene sulfonate (PEDOT:PSS), carbon-based materials, and various types of microparticles and nanoparticles are popular conductive fillers to hydrogel matrices. ${ }^{12-17}$ The mechanical and electrical performance of the conducting hydrogels, however, is usually compromised due to their multicomponent nature. To overcome these limitations, synthetic strategies such as pure PEDOT:PSS hydrogels and covalently bound ECHs have been proposed. $^{18-22}$ Furthermore, the integration of ECH within neural electrodes requires advanced patterning techniques to meet miniaturization requirements for high spatial resolution as well as long-term adhesion to maintain chronic performance. Common patterning methods include dip coating, electrodeposition, inkjet printing, or multistep microfabrication. ${ }^{11,15,18,22,23}$

In this study, we report on the design and processing of a soft electrically conducting hydrogel, based on polyacrylamide (PAAm) and PEDOT:PSS, which is covalently bound and compatible with screen-printing and elastomer-based neurotechnology. PAAm is a well-studied and versatile synthetic biocompatible hydrogel with an easily tunable Young's modulus in the range of $0.1-10^{3} \mathrm{kPa}$, making it an ideal candidate for soft coating development. ${ }^{24,25} \mathrm{We}$ assess the physical, electrochemical, and biological properties of the soft

Received: April 13, 2020

Accepted: June 4, 2020 
A

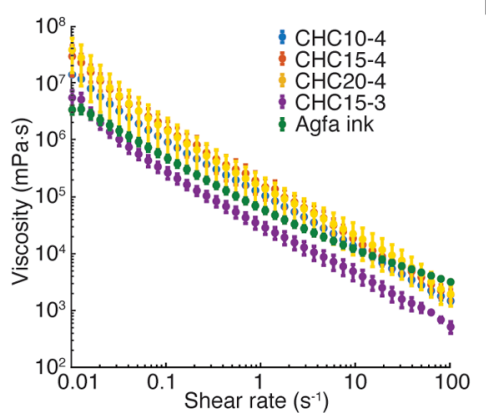

B

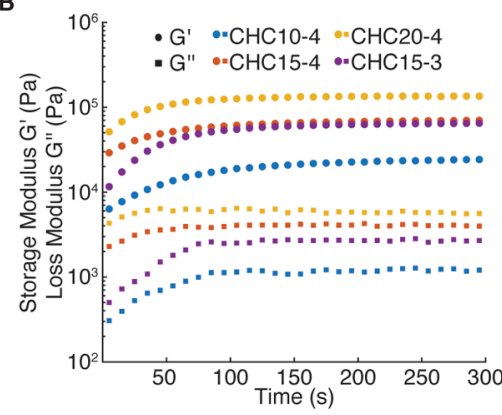

C
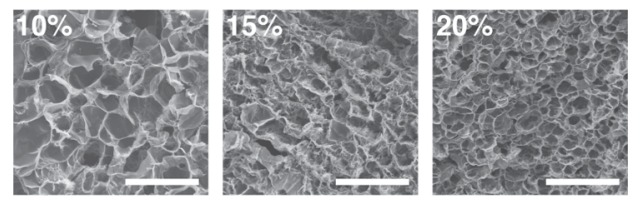

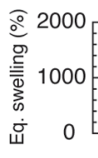
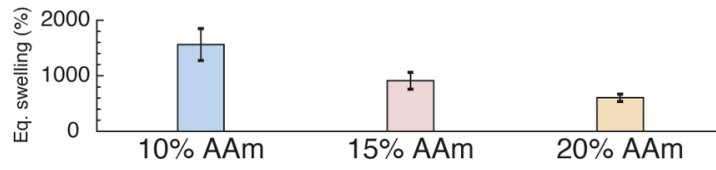

Figure 1. Characterization of the soft conducting hydrogel ink. (A) Viscosity curves of the Agfa ink and CHC formulations with varying \%AAm and \%PEDOT:PSS $(n=3)$. (B) CHC formulation curing kinetics with varying \%AAm and \%PEDOT:PSS. (C) Equilibrium swelling and SEM images of the $\mathrm{CHC}$ with varying AAm concentrations. Scale bar $=20 \mu \mathrm{m}$.

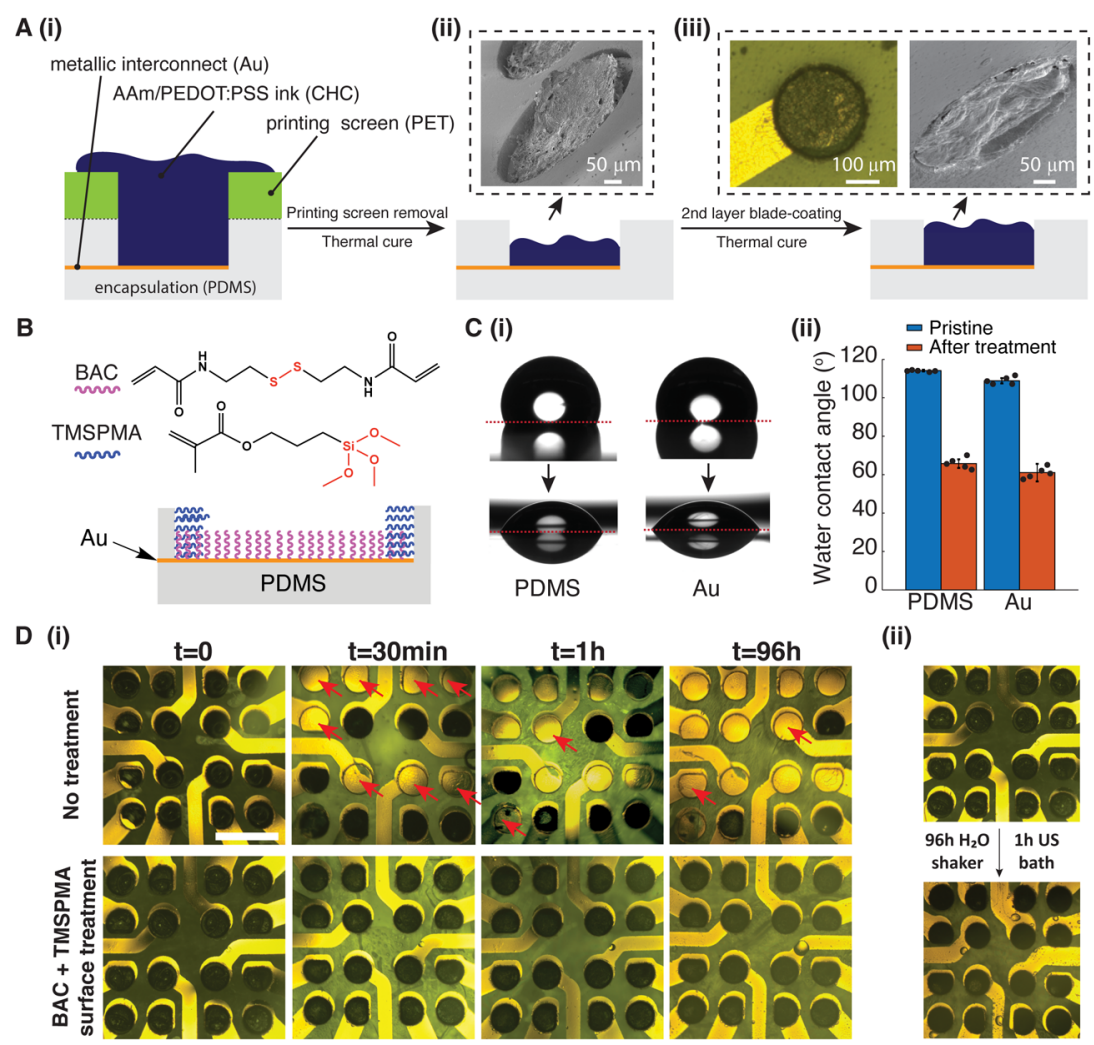

Figure 2. Conducting hydrogel coating patterning and adhesion. (A)(i) Two-step CHC ink patterning process by screen printing and blade coating. (ii) SEM image revealing uneven CHC topography after the first screen-printing step. (iii) Optical and electron microscopy images after the second CHC layer patterning by blade coating. (B) Surface modification agents for PDMS/Au stretchable functional platforms. (C)(i and ii) Water contact angle $(\theta)$ measurements of PDMS and Au before (left) and after (right) surface treatment. Error bars represent one standard deviation $(n=5)$. (D)(i) Water immersion adhesion test of CHC15-4-coated PDMS/Au 16-electrode array with and without surface treatment. Red arrows indicate delaminated electrodes. (ii) Surface-treated array following $96 \mathrm{~h}$ water immersion and $1 \mathrm{~h}$ ultrasonication. Scale bar $=500 \mu \mathrm{m}$.

conductive hydrogel coatings $(\mathrm{CHCs})$ and compare them against those of two other screen-printable coatings: an elastomeric mesocomposite of polydimethylsiloxane/platinum $(\mathrm{PDMS} / \mathrm{Pt}(\mathrm{ElMC}))^{26,27}$ and a commercial, screen-printable, conductive PEDOT:PSS ink (Agfa Orgacon EL-P-5015). We further demonstrate the integration of the $\mathrm{CHC}$ within an elastomeric micro-electrocorticography $(\mu-\mathrm{ECoG})$ array and validate the soft system in acute in vivo recordings while studying its performance and stability.

\section{RESULTS AND DISCUSSION}

Formulation Development and Characterization. The soft CHC formulation is based on mixing an acrylamide
(AAm) monomer solution with a conducting polymer, PEDOT:PSS, to form a viscous dispersion with rheological properties that facilitate printability. The water PEDOT:PSS dispersion structure has been previously described as colloidal core-shell constructs forming a microgel network. ${ }^{28,29}$ An $N, N^{\prime}$-methylenebisacrylamide cross-linker and potassium persulfate thermal initiator are then added to the formulation. In order for a material to be screen printable and therefore categorized as an "ink", two main rheological conditions known as shear thinning and rapid shear recovery should be met. $^{30,31}$ The shear thinning properties of the CHC ink with varying concentrations of the AAm monomer and $\mathrm{PE}$ DOT:PSS were studied while comparing them to those of 
A
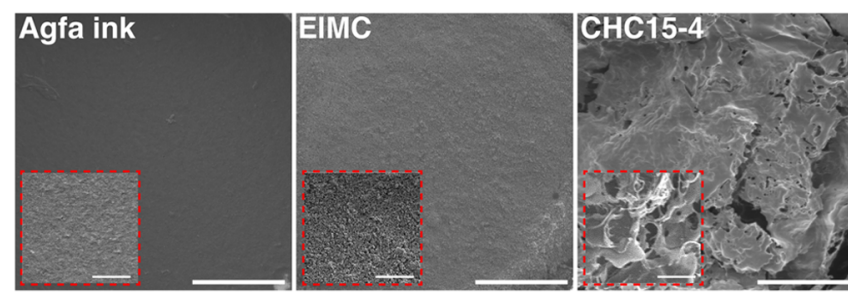

C

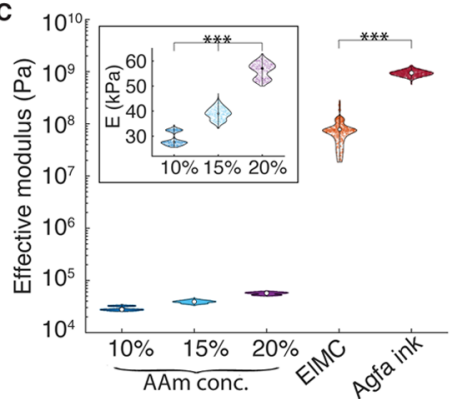

D

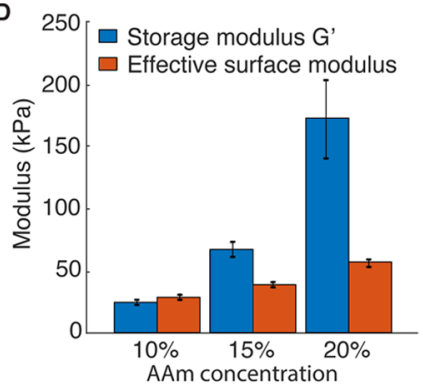

B
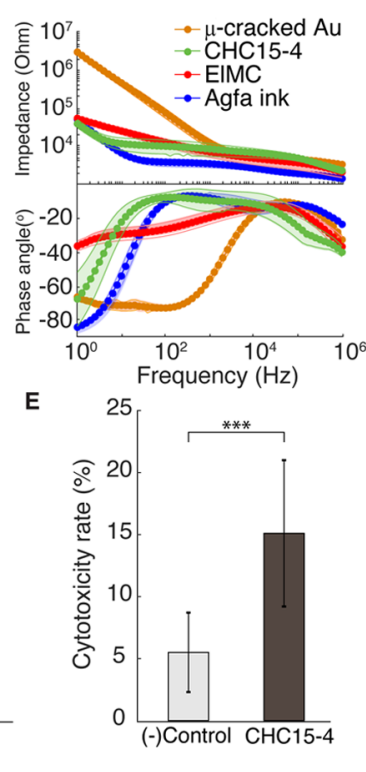

Figure 3. Characterization of different screen-printable electrode coatings. (A) Coating surface SEM images. Scale bar $=50 \mu \mathrm{m}$; inset scale bar $=10$ $\mu \mathrm{m}$. (B) Electrochemical impedance and phase angle spectra of uncoated and coated microcracked Au electrodes $(d=250 \mu \mathrm{m}, n=16)$. (C) Effective elastic modulus computed from AFM nanoindentation measurements as a function of the electrode coating type and CHC composition. Each measurement represents three areas of $16 \times 16 \mu \mathrm{m}^{2}\left(3 \times 250\right.$ points in total). (D) Storage $\left(\mathrm{G}^{\prime}\right)$ and surface effective modulus dependence on \%AAm for CHC compositions. Error bars represent one standard deviation ( $n=3$ for $\mathrm{G}^{\prime}, n=750$ for effective modulus). (E) PC12 cells cytotoxicity rates following $24 \mathrm{~h}$ incubation in a medium containing extracts from $\mathrm{CHC} 15-4$, calculated through viability assay. Error bars represent one standard deviation ( $n=5$ for the negative control and $n=27$ for CHC15-4 samples). $*$ denotes $p<0.05$, $* *$ denotes $p<0.01$, and $* * *$ denotes $p<0.001$.

the Agfa ink as a reference printable material. All CHC formulations exhibited shear thinning-a decrease in viscosity with an increasing shear rate, which is attributed to the PEDOT:PSS domains (Figure 1A). The AAm concentration had no significant effect on the viscosity curves, suggesting no physical interaction between the monomer molecules and the PEDOT:PSS chains. A decreasing PEDOT:PSS concentration, however, resulted in lower viscosity due to lower density of the microgel network. Both the $\mathrm{CHC}$ and Agfa ink showed similar shear thinning trends, in particular at low and medium shear rates, suggesting similar flow characteristics under external shear stress. In addition, both the CHC and Agfa ink exhibited short shear recovery times of 19 and $11 \mathrm{~s}$, respectively, defined as the time required for the storage modulus $\left(G^{\prime}\right)$ to reach $90 \%$ of its initial value following a high shearing event, e.g., printing (Figure S1). Both rheological properties confirmed the printability of the $\mathrm{CHC}$ inks.

Next, the curing kinetics and the $G^{\prime}$ values of the cured formulations were characterized as a function of the AAm monomer concentration while keeping the cross-linker concentration constant $\left(4 \% \mathrm{w} / \mathrm{w}\right.$ AAm). The $\mathrm{G}^{\prime}$ of the cured formulations increased with the AAm concentration $(24.5 \pm 1.7,67.5 \pm 6.8$, and $172.0 \pm 32.4 \mathrm{kPa}$ for 10,15 , and 20\% AAm, respectively) (Figure 1B). The PEDOT:PSS concentration did not affect the $\mathrm{G}^{\prime}$, as previously reported by Feig et al. ${ }^{20}$ This suggests that the PEDOT:PSS domains do not take part in the hydrogel cross-linking process and remain physically entangled within the cured PAAm, forming a semiinterpenetrating network (SIPN) where only one of the constituents is cross-linked. ${ }^{13}$ Furthermore, due to the colloidal nature of the ink, the storage modulus (elastic component) was found to be higher than the loss modulus (viscous component) even in the uncured state. Therefore, no crossover point is observed during the curing process but rather an increase in both the elastic and viscous components of the modulus. Once cured, the $\mathrm{CHC}$ is allowed to swell to equilibrium and is then stored in its fully swollen state for any further characterization or manipulation.

Finally, the swelling of $\mathrm{CHC}$ formulations containing 4\% ( $w / w)$ PEDOT:PSS, which was found to be the solubility limit of the conducting polymer, was characterized with varying monomer concentrations. Bulk CHC samples were cured and allowed to swell to equilibrium then fully dried under vacuum. The weight difference was used to calculate the water uptake and the swelling ratio, which was found to be inversely related to the AAm concentration, as expected. The swelling ratio trend was also consistent with the porosity of the hydrogels, showing a decrease in pore size as the monomer concentration increases (Figure 1C).

The formulation (CHC15-4) consisting of $15 \%(w / w)$ AAm and $4 \%(\mathrm{w} / \mathrm{w})$ PEDOT:PSS was used in all subsequent experiments due to a favorable balance between limited swelling ratios and mechanical properties.

Coating Patterning and Adhesion. The compatibility of the soft $\mathrm{CHC}$ with screen-printing patterning was studied using an entirely stretchable interface design consisting of a PDMS elastomeric substrate and stretchable gold ( $\mathrm{Au})$ interconnects, serving as a functional testing platform (Figure $2 \mathrm{~A}(\mathrm{i}))^{32,33}$ To enhance the adhesion of the $\mathrm{CHC}$ to the substrate, both PDMS and Au surfaces were chemically modified with a monolayer of acryloyl groups. $N, N^{\prime}$ bis(acryloyl)cystamine (BAC) is known to react with $\mathrm{Au}$ through reduction of the disulfide group, forming a thiol-Au bond, ${ }^{34}$ while 3-(trimethoxysilyl)propyl methacrylate (TMSPMA) is a common silanization agent for PDMS (Figure 2B). The surface treatment was performed through a 
polyethylene terephthalate (PET) mask to localize the chemical modifications specifically at the desired electrode sites while preventing any unwanted reactions of the surrounding zones. The surface treatment efficiency was validated by contact angle $(\theta)$ measurements: a decrease from 114 to $66^{\circ}$ and from 109 to $61^{\circ}$ was observed for PDMS and $\mathrm{Au}$ surfaces, respectively, confirming the increased hydrophilicity of the treated surfaces due to the anchored polar acryloyl groups (Figure 2C). Next, CHC15-4 ink was patterned by screen printing through the same PET mask and cured for 15 min at $80{ }^{\circ} \mathrm{C}$ under a cover slip, following removal of the PET mask. A second layer of the CHC15-4 ink was then applied by blade coating and cured to increase the overall coating thickness and compensate for any shrinkage occurring due to cross-linking of the first layer (Figure 2A(ii),A(iii)).

Coating adhesion was qualitatively evaluated by immersing surface-treated $\mathrm{CHC} 15$-4-coated samples $(n=2,16 \mathrm{Au}$ electrodes each) in deionized (DI) water for $96 \mathrm{~h}$ followed by $1 \mathrm{~h}$ of ultrasonication. Both samples remained completely intact without any noticeable delamination of the coating. A control sample (without surface treatment) exhibited significant delamination of $75 \%$ (12 out of 16) of the coated electrode sites after $96 \mathrm{~h}$ of water immersion (Figure 2D). Despite these encouraging results, further characterization is required to assess the long-term adhesion and stability of the coating for potential chronic applications.

Mechanical and Electrochemical Coating Characterization. First, the surface morphology of conductive electrode coatings was studied (Figure 3A). The Agfa coating was found to be smooth, without any remarkable topographical features. The EIMC coating, on the other hand, was characterized by a high microroughness, originating from the Pt microparticles embedded inside the PDMS matrix. CHC-15-coated samples exhibited a porous structure, as expected from a hydrogel matrix.

However, since the CHC sample was lyophilized prior to imaging in the SEM, its original surface morphology has been altered due to partial collapse of the pores. Electrochemical performance of the conductive hydrogel coating and the reference coatings was evaluated using electrochemical impedance spectroscopy (EIS) and cyclic voltammetry (CV). All conductive coatings displayed similar impedance profiles, reducing the modulus value compared to the bare $\mathrm{Au}$ electrodes (Figure 3B). Lower impedances of the coated electrodes are expected to improve the signal-to-noise ratio (SNR) by screening thermal noise and shunting effects. ${ }^{35}$ Cathodic charge storage capacity $\left(\operatorname{CSC}_{\mathrm{c}}\right)$ values, which provide information on availability of the electrochemical surface area for current transfer, were found to be lower for $\mathrm{CHC} 15-4$ - and Agfa-coated electrodes $\left(13.9 \pm 4.6\right.$ and $11.1 \pm 1.5 \mathrm{mC} \mathrm{cm}^{-2}$, respectively) compared to ElMC-coated ones $(38.1 \pm 4.0 \mathrm{mC}$ $\mathrm{cm}^{-2}$ ) (Figure S2). CSC $\mathrm{c}_{\mathrm{c}}$ values are known to be influenced by factors such as surface roughness, electrochemical surface area (ESA), and charge transfer mechanisms (ionic vs electronic or both) of the coating. ${ }^{36}$ The significantly higher $\operatorname{CSC}_{\mathrm{c}}$ values of the ElMC-coated devices are attributed to the coating's high microroughness as compared to the porous $\mathrm{CHC}$ and much smoother Agfa coatings. The microstructured surface increases the ESA of the coating. Although a relatively high $C C_{c}$ value for CHC-coated electrodes was expected due to the large ESA of the porous hydrogel, it was found to only slightly exceed that of the Agfa coating. We assume that this might be due to a non-optimal concentration and incorporation of the conduct- ing polymer within the PAAm matrix during its polymerization caused by miscibility differences as reported for other electrically conducting hydrogels. ${ }^{13}$ A higher PEDOT:PSS concentration, however, could not be achieved due to miscibility limitations. Next, the surface mechanical properties of CHC-coated electrodes with different monomer concentrations were characterized using AFM nanoindentation and compared to those of ElMC- and Agfa-coated electrodes. Surface mechanical properties of hydrogels tend to vary from bulk properties due to hydration gradients and surface inhibition during free radical polymerization and cross-linking reactions as well as surface defects. ${ }^{25,37} \mathrm{CHC}$-coated samples were immersed in DI water to maintain swelling equilibrium then indented using a soft cantilever $\left(0.12 \mathrm{~N} \mathrm{~m}^{-1}\right)$ modified with a polystyrene bead (PS, $4.5 \mu \mathrm{m}$ diameter) to allow a larger probing area due to hydrogel porosity and topography. Agfaand ElMC-coated samples were indented in air using a stiff cantilever $\left(40 \mathrm{~N} \mathrm{~m}^{-1}\right)$. The approach curves were used to calculate the effective elastic modulus by applying the Hertz and Sneddon models for the soft and stiff cantilevers, respectively (Figure S3). As expected, the effective elastic modulus of the $\mathrm{CHC}$ increased with the monomer concentration $(28.8 \pm 2.5,39.0 \pm 2.4$, and $56.3 \pm 3.0 \mathrm{kPa}$ for 10,15 , and $20 \% \mathrm{AAm}$, respectively). Furthermore, the effective modulus of the CHC-coated samples was found to be five to six orders of magnitude lower than that of ElMC- and Agfa-coated samples $(90.9 \pm 29.4 \mathrm{MPa}$ and $2.3 \pm 0.6 \mathrm{GPa}$, respectively) (Figure 3C). It is worth noting that the surface effective modulus was found to be less influenced by \%AAm compared to the storage modulus (Figure 3D). Although increasing the \%AAm from 10 to 15 and $20 \%$ resulted each time in about 2.5 times increase in $\mathrm{G}^{\prime}$, the surface effective modulus increase was only by a factor of 1.4. We assume this might be due to oxygen surface inhibition during the free radical polymerization of PAAm. Although the screen-printed $\mathrm{CHC}$ is cured under a glass slip, the sealing is not hermetic, and oxygen might be still present at the surface, reducing the cross-linking density and resulting in a softer layer close to the surface. $^{38}$ The storage modulus, however, is a bulk property; therefore, it is less sensitive to surface inhibition. Oxygen inhibition in the bulk exists in both cases since the formulation cannot be efficiently purged to remove dissolved oxygen due to its high viscosity and the patterning process during which it is exposed to an ambient environment. Furthermore, the AFM measurements were performed on fully swollen $\mathrm{CHC}$ samples, whereas the $G^{\prime}$ values represent swelling that is lower than equilibrium, therefore resulting in higher stiffness. Overall, the CHC mechanical properties are in good agreement with previously reported results of similar formulations, showing that screen printing as a patterning technique does not affect the final mechanical properties of the soft material. ${ }^{20,39}$

In Vitro Toxicity and Cell Attachment. An in vitro cytotoxicity test, assessing in particular the potential toxicity of the AAm monomer, ${ }^{40,41}$ was performed using bulk samples of CHC15-4 (geometrical surface area: $1.5 \mathrm{~cm}^{2}$, volume: 0.14 $\mathrm{cm}^{3}$; samples were washed for $24 \mathrm{~h}$ to remove excess reactants). Since the surface area of each sample is almost 200 times higher and its volume is 3500 times larger, compared to the coating material used for an entire 16-electrode array with $250 \mu \mathrm{m}$ sites, the experiment amplifies any toxic effects originating from the formulation. Samples were sterilized and soaked in a cell culture medium during $24 \mathrm{~h}$ to facilitate the extraction of any excess reactants or other components. The 
A
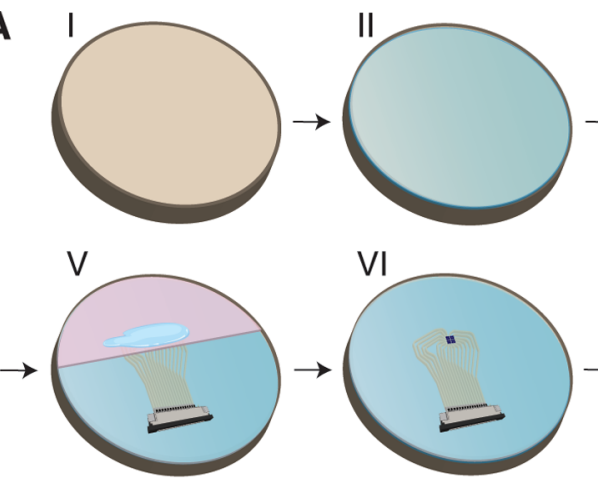

C

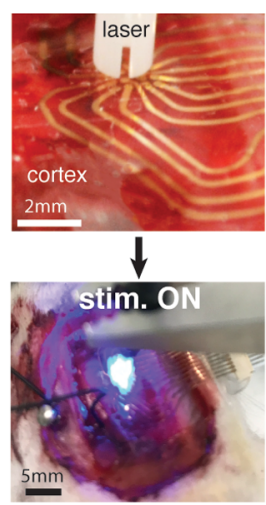

F (i)

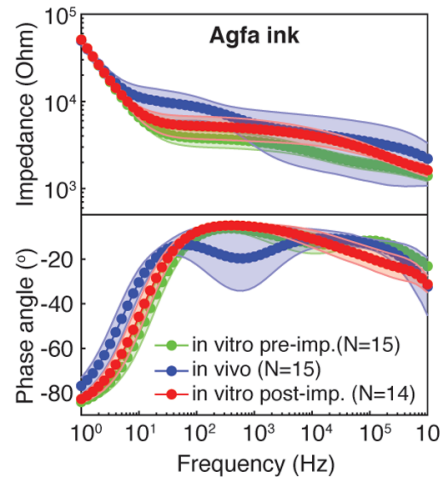

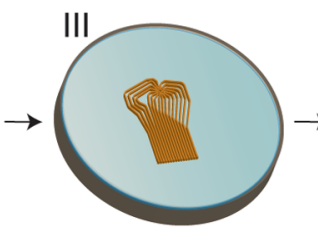

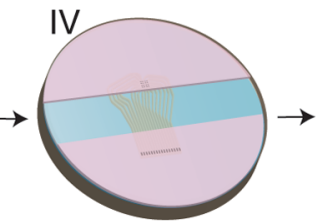

VII

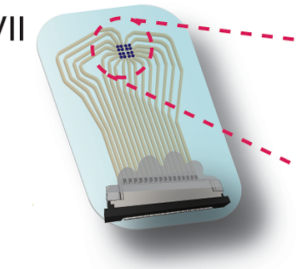

D

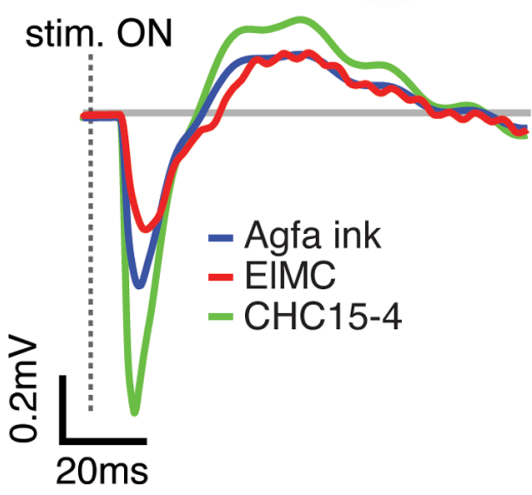

B

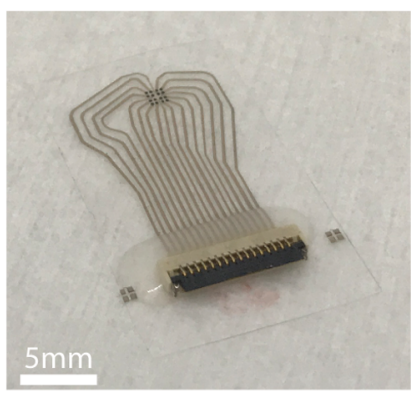

E

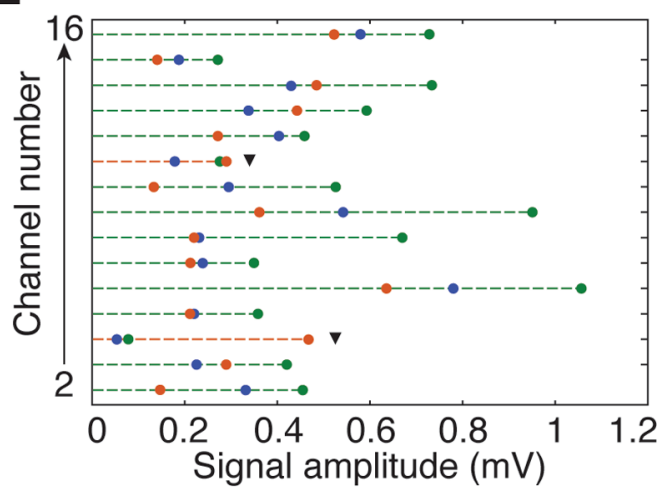

(ii)

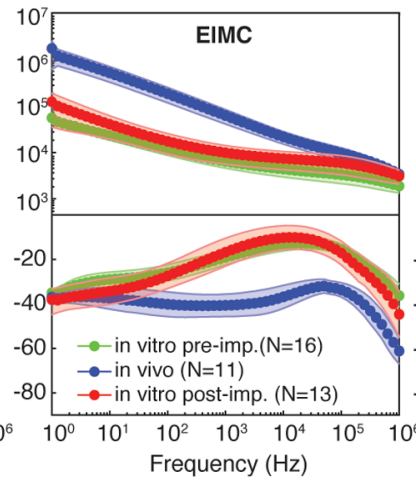

(iii)

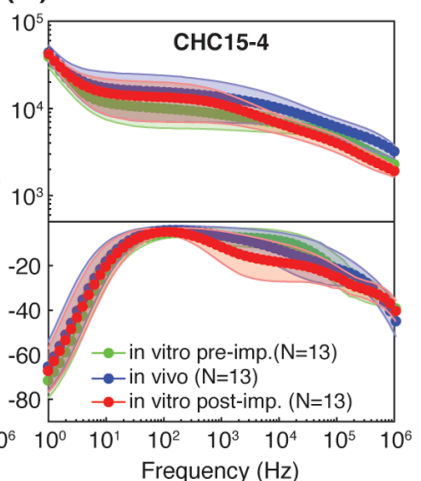

G

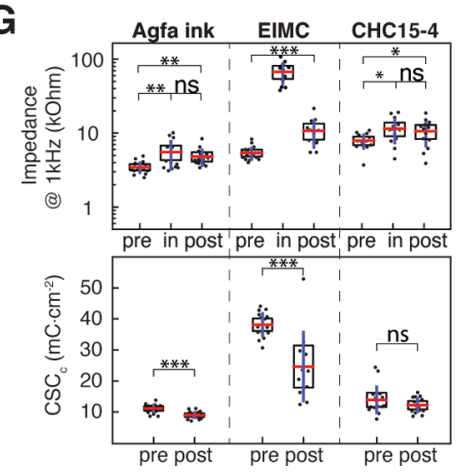

Figure 4. Fabrication and in vivo characterization of soft and conformable $\mu$-ECoG array. (A) Schematic of the array process flow. (I) Dextran release layer spin coating. (II) PDMS substrate spin coating. (III) $\mathrm{Cr} / \mathrm{Au}$ thermal evaporation through a shadow mask. (IV) Plasma bonding of a precut PDMS-PET encapsulation stack. (V) Ag paste screen printing and ZIF connector mounting. PDMS and Au surface treatment using BAC/ TMSPMA (for the CHC only). (VI) Electrode coating screen printing and curing. (VII) Array release and cutting. (B) CHC15-4-coated $\mu$-ECoG array. (C) Optical fiber positioning over the center of a $\mu$-ECoG array placed on the surface of a Thy1-COP4/YFP rat cortex. (D) Representative signal and (E) signal amplitudes of different coatings showing higher signals for CHC15-4-coated electrodes (blue, Agfa-coated electrodes; red, ElMC-coated electrodes; green, CHC15-4-coated electrodes). Signals were averaged over 120 optical pulses $(2 \mathrm{~Hz}, 5 \mathrm{~ms}, 50 \mathrm{~mW}$ optical power). The color of the dashed line corresponds to the coating with the highest signal amplitude. The solid inverted triangle symbol denotes channels in which the CHC15-4 coating did not generate the highest signal amplitude. (F) Electrical impedance and phase angle spectra for different coatings measured before, during, and after acute implantation. (i) Agfa ink-, (ii) ElMC-, and (iii) CHC15-4-coated arrays. Preimplantation and postimplantation spectra were acquired in PBS, and intraoperative spectra were acquired by using a needle pierced through the skin as ground and reference electrodes. (G) Electrical impedance values at $1 \mathrm{kHz}$ and $\mathrm{CSC}_{\mathrm{c}}$ comparison for different coatings before, during (EI only), and after acute implantation. Data is presented as the mean (red bar), standard error (blue bar), and $95 \%$ confidence level (box). $*$ denotes $p<0.05$, $* *$ denotes $p$ $<0.01$, and $* * *$ denotes $p<0.001$.

medium containing the extracts was then transferred into a well plate, which was preseeded with fluorescent pheochromocytoma neuronal cells (PC12). Following a $24 \mathrm{~h}$ incubation period, a live/dead viability assay revealed a low cytotoxicity rate of $15.1 \pm 5.9 \%$ of the $\mathrm{CHC}$ (Figure 3E). A cell culture medium $(5.5 \pm 3.2 \%$ cytotoxicity rate $)$ and lysis buffer $(100 \%$ cytotoxicity rate) were used as negative and positive controls, respectively. Then, the neuronal cell attachment and neurite outgrowth were evaluated on various coatings. The coatings were patterned on 16-electrode elastomeric PDMS/Au arrays subsequently coated with collagen-I for initial cell adhesion. Efficient attachment of the collagen on the different arrays was verified using SEM (Figure S4). Following cell seeding and a $24 \mathrm{~h}$ proliferation period, a differentiation medium containing 
a nerve growth factor (NGF) was introduced to promote neurite outgrowth. After 5 days in vitro, no cell attachment was observed on the Agfa- and ElMC-coated electrodes whilst more than $60 \%$ of the $\mathrm{CHC} 15$-4-coated electrodes had cells attached as well as extensive neurite outgrowth (based on $n=$ 6). Although all electrode arrays were initially coated with collagen-I to promote cell adhesion, following the 5 day incubation and numerous medium changes, cells were found to only attach to the CHC15-4-coated electrodes (Figure S5). It should be noted that the studied electrode coatings differ not only in morphology and mechanical properties but also in their chemical surface properties, such as polarity and hydrophilicity $\left(\theta=80.0 \pm 5.6\right.$ and $100.8 \pm 5.3^{\circ}$ for Agfa and ElMC, respectively) - all known to be key factors for cell attachment and robust neurite outgrowth. ${ }^{42-44}$ Considerable cell attachment and neurite outgrowth were also observed on the PDMS encapsulation around the electrode sites on all samples and on bare gold electrodes, ${ }^{45}$ confirming the overall cell viability and normal differentiation patterns.

These initial observations of neural cell adhesion and neurite outgrowth on the $\mathrm{CHC}$-coated electrodes are encouraging cues for future implantable neural interfaces. As close contact between neurons and the electrode site is essential for an efficient electrical charge transfer for neural recording purposes, the coating's low toxicity and the neural cells' high affinity toward it are promising indications of its biocompatibility.

Soft MEA Manufacturing and In Vivo Recording. The soft $\mathrm{CHC}$ was integrated within the fabrication process flow of an elastomeric $\mu$-ECoG array. Sixteen-channel arrays were microfabricated using thin-film and silicone processing technology inspired from the e-dura process. ${ }^{26,33}$ The $\mathrm{CHC}$ integration included a BAC-TMSPMA surface treatment of the substrate and a two-step ink patterning by screen printing and blade coating followed by thermal curing (Figure 4A). Once cured, the $\mathrm{CHC}$ was swollen and kept hydrated to prevent any shrinkage-related stresses due to drying. The device final thickness was $100 \mu \mathrm{m}$ to offer both good surgical handling and conformability to the epidural surface of the cortex. Each electrode was $250 \mu \mathrm{m}$ in diameter (Figure 4B). Reference arrays coated with ElMC and Agfa ink were fabricated using a similar process. A preliminary acute recording experiment was performed in a Thy1-COP4/YFP rat that expresses the Channelrhodopsin-2 light-activated ion channels in pyramidal neurons of the cerebral cortex. ${ }^{46}$ After performing a craniotomy under urethane anesthesia, each of the tested $\mu$-ECoG arrays was epidurally placed over the rat's cortex (maintaining the same orientation for all arrays). A blue laser beam $\left(\lambda=473 \mathrm{~nm}\right.$, power density $\left.=1.6 \mathrm{~W} \mathrm{~mm}^{-2}\right)$ was directed to the center of the array to optically activate cortical neurons (Figure 4C).

The optical stimulation led to electrocorticogram responses that were reliably recorded from the arrays ( 120 pulses, $2 \mathrm{~Hz}, 5$ ms pulse width, $50 \mathrm{~mW}$ optical power; Figure 4D and Figure S6). Interestingly, the amplitude of the signals acquired using a CHC15-4-coated array was significantly higher (up to almost $300 \%)$ compared to all of the 15 signals obtained from the Agfa-coated array $(p<0.05)$. Similar difference in signal amplitude was observed for 13 out of the 15 channels of the CHC15-4-coated array compared to the ElMC-coated device (Figure 4E). Noise levels were found to be low for all of the devices, not exceeding $2 \mu \mathrm{V}$ (Figure S7). A control experiment using a wild-type rat was performed to exclude any signal generation due to nonphysiological currents caused by a photoelectric effect. ${ }^{47}$

By comparing the electrochemical properties (EIS and $C S C_{c}$ ) before, during, and after the acute implantation, we were able to assess the stability and robustness of the different coatings. ElMC-coated arrays were found to be particularly sensitive to in vivo conditions. Not only their impedance spectra significantly differed from the in vitro measurements, showing a more capacitive behavior and a higher impedance at $1 \mathrm{kHz}$ but also their $C_{\mathrm{c}}$ dropped dramatically postimplantation (Figure 4F,G). The impedance of the Agfa- and CHC15-4-coated devices, on the other hand, showed only a slight increase when measured in vivo and remained stable postimplantation. Moreover, unlike the Agfa-coated devices, which exhibited a drop in $C_{\mathcal{c}}$, comparable $C_{\mathcal{c}} C_{\mathrm{c}}$ values were measured for the $\mathrm{CHC} 15-4$ coating before and after the acute implantation, suggesting negligible chemical and physical changes occurring at the coating surface. These variations in the electrochemical performance may be attributed to differences in the surface properties of the coatings. Since both Agfa and ElMC coatings are hydrophobic, intimate electrode-tissue interfacing is limited due to poor wettability of the electrode surface by the cerebrospinal fluid (CSF). Furthermore, we hypothesize that the changes in $C_{C S}$ following the acute implantation might be also linked to the fouling properties of the coating materials. While it is known that PAAm has antifouling properties that limit protein adsorption, PDMS has high affinity toward those biomolecules. ${ }^{48-50}$ The matrix of the commercial Agfa ink is unknown; therefore, its fouling properties cannot be assessed. Adsorption of proteins originating from the CSF and plasma while in contact with the electrodes at the implantation site might alter charge transfer mechanisms at the coating interface, impacting impedance and $C_{S C}$. These preliminary recording results together with the in vitro stability of the electrochemical properties of the soft coating suggest the printable $\mathrm{CHC}$ can find applications as an efficient transducing material to monitor neural signals. This entirely soft electrode array offers recording capability acutely on par with state-of-the-art PEDOT:PSS-based devices. Further in vivo and chronic evaluations are now needed to confirm the potential of this soft conducting material for biointegrated implants.

\section{CONCLUSIONS}

In summary, a screen-printable PAAm-PEDOT:PSS conductive hydrogel gathers several of the critical mechanical and electrochemical features of materials for electrode coatings. Screen-printing patterning is straight forward and compatible with a wide range of carrier materials. Further work will include chronic evaluation of coated arrays and assessment of their long-term performance and biointegration, which may open new opportunities for implantable bioelectronic devices in particular for chronic neural applications.

\section{EXPERIMENTAL SECTION}

All chemicals were purchased from Sigma-Aldrich unless specified otherwise.

AAm/PEDOT:PSS (CHC) Ink Formulation. Conducting polymer aqueous dispersions of different concentrations were prepared by dissolving 3 and 4 wt \% PEDOT:PSS (Orgacon dry PEDOT:PSS) in DI water upon vigorous stirring for $48 \mathrm{~h}$ at $70{ }^{\circ} \mathrm{C}$. An acrylamide monomer $\left(53,85\right.$, and $120 \mathrm{mg}$ for 10,15 , and $20 \%\left(\mathrm{w} / \mathrm{w} \mathrm{H}_{2} \mathrm{O}\right)$, respectively) was then added to $0.5 \mathrm{~g}$ of the PEDOT:PSS dispersion. 
The cross-linker, $N, N^{\prime}$-methylenebisacrylamide (4\% (w/w AAm)), and potassium persulfate thermo-initiator $(4 \mathrm{mg})$ were finally added, and the formulation was manually mixed before patterning.

Ink Rheological Characterization. All measurements were performed using a rheometer (MCR 302, Anton-Paar) equipped with a Peltier heating system and a cone-plate geometry $\left(1^{\circ}, 25 \mathrm{~mm}\right.$ cone, $40 \mathrm{~mm}$ plate, sand-blasted). Viscosity curves were obtained at $25^{\circ} \mathrm{C}$. Curing kinetics were measured by first equilibrating the sample for $5 \mathrm{~min}$ at $25^{\circ} \mathrm{C}$ followed by a $3{ }^{\circ} \mathrm{C} \mathrm{min}^{-1}$ ramp until reaching 80 ${ }^{\circ} \mathrm{C}$ and maintaining a $5 \mathrm{~min}$ isotherm. Measurements were taken at 1 $\mathrm{Hz}$ with $1 \%$ amplitude. Shear recovery was performed by subjecting the sample to alternating intervals of low and high shear rates (vibrational deformation of $1 \%$ at $1 \mathrm{~Hz}$ for $30 \mathrm{~s}$, a high shearing event at $100 \mathrm{~s}^{-1}$ for $1 \mathrm{~s}$, followed by vibrational deformation of $1 \%$ at $1 \mathrm{~Hz}$ for another $60 \mathrm{~s}$ ). The $\mathrm{G}^{\prime}$ recovery time was calculated as the time required to reach $90 \%$ of its initial value following the second interval.

Swelling Tests. Cured bulk CHC samples of different \%AAm were allowed to swell to equilibrium. The samples were then gently blotted, and their weight was recorded $\left(W_{\mathrm{s}}\right)$. The swollen samples were dried at $100{ }^{\circ} \mathrm{C}$ under vacuum and weighted again $\left(W_{\mathrm{d}}\right)$. The swelling ratio was calculated using the following equation: \% swelling $=100 \cdot\left(W_{\mathrm{s}}-W_{\mathrm{d}}\right) / W_{\mathrm{d}}$. A total of 12 specimens were measured for each formulation.

Electron Microscopy. All SEM images were acquired in high vacuum mode without any additional coating (SU5000, Hitachi). $\mathrm{CHC}$ - and collagen-coated samples were lyophilized prior to imaging.

Surface Treatment of Au/PDMS. A $23 \mu \mathrm{m}$ PET shadow mask was laminated on a Si wafer with Au and PDMS features followed by oxygen plasma activation $\left(20 \mathrm{~W}, 30 \mathrm{~s}, 0.5 \mathrm{mbar}_{2}\right.$ ). A $3 \mathrm{~mL} \mathrm{~N}, \mathrm{~N}^{\prime}$ bis(acryloyl)cystamine (Thermo Fisher Scientific) solution (10 nM in methanol) was mixed with a $3 \mathrm{~mL}$ solution of 3-(trimethoxysilyl)propyl methacrylate $(1: 20(\mathrm{v} / \mathrm{v})$ in ethanol).A few drops of the mixture were placed over the exposed Au features on the wafer and left for $90 \mathrm{~min}$ in the dark, adding a few more drops every $15 \mathrm{~min}$ to prevent drying. Then, the wafer was rinsed with isopropanol and dried under a nitrogen flow.

Contact Angle Measurements. Water contact angle measurements were performed using the sessile drop method (DSA-30E, Krüss).

AAm/PEDOT:PSS (CHC) Screen Printing. The CHC ink was manually screen printed through a $23 \mu \mathrm{m}$ thick PET printing screen which was laminated on top of a PDMS substrate, using a glass slide as a blade. Then, the PET screen was removed, and the ink was covered with a glass slip and cured for $15 \mathrm{~min}$ on an $80^{\circ} \mathrm{C}$ hot plate. A second layer of the ink was blade coated using a glass slide as a blade and cured again for $15 \mathrm{~min}$ on an $80^{\circ} \mathrm{C}$ hot plate covered with a glass slip. The cured coating was then rehydrated in DI water.

Reference Coating Formulations. Elastomeric PDMS/Pt mesocomposite (ElMC) was prepared as previously described. ${ }^{26}$ Briefly, Pt microparticles (average size: 0.27-0.47 $\mu \mathrm{m}$, Strem Chemicals) were mixed with PDMS (Sylgard 184, Dow Corning) at $70 \%(\mathrm{w} / \mathrm{w})$. Cyclohexane $(2: 1 \mathrm{w} / \mathrm{w})$ was added to the formulation to decrease the viscosity and facilitate screen printing.

Commercial PEDOT:PSS screen-printable ink (Agfa Orgacon EL$\mathrm{P}$-5015) was used as received.

Both were manually screen printed through a $23 \mu$ m-thick PET printing screen which was laminated on top of a PDMS substrate, using a glass slide as a blade. Then, the PET screen was removed and the coatings were thermally cured. The ElMC coating was cured overnight at RT followed by $4 \mathrm{~h}$ at $55{ }^{\circ} \mathrm{C}$. The Agfa ink was cured at $80{ }^{\circ} \mathrm{C}$ for $20 \mathrm{~min}$.

Adhesion Test. Coated and uncoated samples were immersed in DI water and placed on an orbital shaker (150 rpm, KS250basic, IKA) for $96 \mathrm{~h}$ followed by $1 \mathrm{~h}$ of ultrasonication $(40 \mathrm{kHz}, 130 \mathrm{~W})$ at RT.

Electrochemical Characterization. All measurements were performed in PBS using a potentiostat $(600+$, Gamry Instruments) in a three-electrode setup. For in vitro measurements, a platinum wire serving as the counter electrode and $\mathrm{Ag} / \mathrm{AgCl}$ electrode as the reference electrode were used. For in vivo measurements, a needle pierced through the skin was used as counter and reference electrodes.
Electrochemical impedance spectroscopy was performed in the range of $1 \mathrm{~Hz}-1 \mathrm{MHz}$. Cyclic voltammetry was performed between -0.6 and $0.8 \mathrm{~V}$ at a scan rate of $0.1 \mathrm{~V} \mathrm{~s}^{-1}$. Cathodic charge storage capacity $\left(\operatorname{CSC}_{\mathrm{c}}\right)$ was calculated from the time integral of the cathodic current.

All electrodes were activated prior to characterization by performing 20 fast $\mathrm{CV}$ scans at a $100 \mathrm{~V} \mathrm{~s}^{-1}$ scan rate.

Nanomechanical Characterization. All measurements were performed with AFM in QNM mode (Dimension Icon, Bruker) with different cantilevers. A silicon nitride $(\mathrm{SiN})$ cantilever modified with a $4.5 \mu \mathrm{m}$-dimeter polystyrene bead $\left(0.12 \mathrm{~N} \mathrm{~m}^{-1}\right.$, Novascan $)$ was used for the CHC coatings. A Si cantilever was used for both ElMC and Agfa coatings (40 N m${ }^{-1}$, RTESPA-300-30, Bruker). CHC samples were probed in DI water to ensure swelling to equilibrium of the coating, whereas the ElMC and Agfa coating samples were measured in air. Each sample was probed at three different areas of 16 $\times 16 \mu \mathrm{m}^{2}$ with a $1 \mu \mathrm{m}$ step size. The approach curves were used to calculate the effective modulus by fitting the curves using the Hertz model for the SiN-PS spherical cantilever and Sneddon model for the $\mathrm{Si}$ conical cantilever.

Cytotoxicity and Neurite Outgrowth. All cell culture products were purchased from Gibco unless specified otherwise.

For cytotoxicity studies, three sample batches were prepared by curing $\mathrm{CHC} 15-4$ ink for $30 \mathrm{~min}$ at $80{ }^{\circ} \mathrm{C}$ inside a cylindrical mold. After demolding, each sample was hydrated, cut into three discs (surface area: $1.5 \mathrm{~cm}^{2}$, volume: $0.14 \mathrm{~cm}^{3}$ ), and ozone sterilized (SteriVet, Sterilux). The sterile samples $(n=9)$ were then soaked in $1.5 \mathrm{~mL}$ of a proliferation culture medium composed of RPMI-1640 GlutaMAX (61870-010) supplemented with $10 \%$ heat-inactivated horse serum (16050-122), 5\% fetal bovine serum (10270-106), and Geneticin $\left(250 \mu \mathrm{g} \mathrm{mL}^{-1}, 10,131-019\right)$, and left for $24 \mathrm{~h}$ to allow extraction of CHC components. In parallel, green fluorescent PC12 cells (Linterna P20109, Innoprot) were seeded on a collagen-I-coated 48-well plate at a density of $2 \cdot 10^{4}$ cells $\mathrm{cm}^{-2}$ in $0.5 \mathrm{~mL}$ of the proliferation medium. Following a $24 \mathrm{~h}$ incubation phase, the medium containing nonattached cells was removed and replaced with $0.5 \mathrm{~mL}$ of the conditioned medium, which was in contact with the $\mathrm{CHC}$ samples (three wells per CHC sample, 27 wells in total). A sterile medium was added to wells used as positive and negative controls $(n$ $=5$ for each). After another $24 \mathrm{~h}, 4 \mu \mathrm{L}$ of lysis buffer (G1828, Promega) was added to the positive control wells. Then, propidium iodide (5 mg mL $\mathrm{m}^{-1}, 81845$, Fluka) and Hoescht dye $\left(4 \mathrm{mg} \mathrm{mL}^{-1}\right.$, $\mathrm{H} 3570$, Invitrogen) were added to each well and incubated for $20 \mathrm{~min}$ before imaging with a fluorescence microscope (DMI4000B, Leica). Quantitative analysis of dead and total cells was performed using Fiji/ ImageJ software. The cytotoxicity rate was calculated as the ratio between dead cells (propidium iodide labeled) and total cells (Hoescht labeled). Results are presented as the average between all the 27 wells for the CHC samples and as the average between five wells for the negative and positive controls ( $100 \%$ cytotoxicity rate).

The neural cell adhesion and neurite outgrowth study was performed using a similar protocol. Elastomeric PDMS/Au 16electrode array samples (Agfa, ElMC and CHC15-4-coated) were ozone-sterilized in a 12-well plate with one sample per well. Each sample was coated with collagen-I $\left(150 \mu \mathrm{g} \mathrm{mL} \mathrm{mL}^{-1}\right.$ diluted in PBS, A10483-01) and incubated for $1 \mathrm{~h}$ at $37^{\circ} \mathrm{C}$. The remaining collagen-I solution was removed and $4 \times 10^{4}$ green fluorescent PC12 cells were seeded onto the sample to cover the entire 16 electrode sites. After 30 min, a proliferation medium was added as described earlier. Following a $48 \mathrm{~h}$ incubation, the medium was changed to a differentiation medium containing an Opti-MEM medium (31985-047) supplemented with $0.5 \%$ fetal bovine serum and a nerve growth factor (NGF, $\left.50 \mathrm{ng} \mathrm{mL} \mathrm{m}^{-1}, 13257-019\right)$ to promote neurite outgrowth as described by $\mathrm{Hu}$ et al. ${ }^{51}$ Following 5 days of incubation, the samples were imaged with a confocal fluorescent microscope to observe cell adhesion and neurite outgrowth.

$\boldsymbol{\mu}$-ECoG Device Fabrication. First, a Si wafer was oxygen plasma activated $\left(20 \mathrm{~W}, 30 \mathrm{~s}, 0.5 \mathrm{mbar}_{2}\right)$ followed by spin coating of $20 \mathrm{wt}$ $\%$ dextran solution in DI water at $2000 \mathrm{rpm}$ and drying for $2 \mathrm{~min}$ at $120{ }^{\circ} \mathrm{C}$. A $50 \mu \mathrm{m}$ PDMS layer (Sylgard 184, Dow Corning, 1:10 mixing ratio) was spin coated and cured at $80{ }^{\circ} \mathrm{C}$ for $3 \mathrm{~h}$ on which a 
precut shadow mask of $23 \mu$ m-thick PET sheet was laminated. Cr/Au $(5 / 35 \mathrm{~nm})$ evaporation through the shadow mask was performed to deposit microcracked $\mathrm{Au}$ interconnects (Auto 306, Edwards). An encapsulation PET-PDMS-PET stack (23-50-23 $\mu \mathrm{m}$ thick) was prepared by PDMS spin coating and curing on a PET sheet followed by a top PET sheet lamination, then electrode and connector pad openings were laser cut. Then, one of the PET layers was removed, and the PDMS encapsulation was plasma bonded to the PDMS-Au wafer. Silver-epoxy paste (H27D Part A, Epoxy Technology) was screen printed on the connector pads; the PET screen mask was removed (around the connector pads only); and a ZIF connector was surface mounted, sealed with RTV (DC 734 RTV clear, Dow Corning), and cured overnight at RT. The BAC/TMSPMA surface treatment solution was then applied on the electrode sites according to the described procedure followed by coating patterning and curing. Conductive hydrogel coatings were applied and cured as described above. ElMC and Agfa coatings were screen printed and cured without prior surface treatment. $\mu$-ECoG devices were released from the wafer by immersion in DI water and dissolution of the sacrificial dextran layer. Devices were then dried at RT, cut, and wired to a flex cable.

Acute Recording Experiments. All animal experiments were approved by the Veterinary Office of the canton of Geneva in Switzerland and were in compliance with all relevant ethical regulations. A W-Tg(Thyl-COP4/YFP) (NBRP 0685, Kyoto, Japan) transgenic female adult rat $(\sim 220 \mathrm{~g}$ body weight $)$ was anesthetized with urethane $\left(1.25 \mathrm{~g} \mathrm{~kg}^{-1}\right.$ body weight, dilution with saline) before being head-fixed in a stereotaxic frame (David Kopf Instruments). A large craniotomy was performed, and a $\mu$-ECoG array was then placed epidurally over the exposed cortex. The array was connected using a flex cable to a custom-made ZIF-Omnetics converter, which was then connected to a pre-amplifier through a Zifclip headstage adaptor (ZCA-OMN32, TDT). A diode-pumped solidstate blue laser (473 nm, Laserglow Technologies) coupled via a FC/ PC terminal connected to a $200 \mu \mathrm{m}$ core optical fiber (ThorLabs) was used to deliver optical stimulation. Using a micromanipulator, the fiber was placed at the center of each $\mu$-ECoG array. A silver ground wire was fixed to the skull using a metallic screw. A second silver wire was placed between the dura and the skull and was used as a reference for differential amplification. Optical stimulation was delivered through the transparent elastomeric array to stimulate the surface of the cortex. A train of light pulses was delivered at $2 \mathrm{~Hz}(5 \mathrm{~ms}$ duration) varying the intensity from 35 to $90 \mathrm{~mW}$. Differential recordings triggered by the optical stimulation were performed using a customized code implemented in a TDT RZ2 system (Tucker Davis Technologies), amplified with a PZ5 pre-amplifier, sampled at 25 $\mathrm{kHz}$, and digital band-passed filtered $(0.1-5000 \mathrm{~Hz})$. Recorded signals were averaged over each individual optical pulse across 120 pulses. Noise levels were recorded during $60 \mathrm{~s}$ at rest without optical stimulation and calculated as the root mean square of the signals.

The procedure was repeated for each of the three $\mu$-ECoG arrays with the different coatings, all maintaining the same orientation on the cortex.

A control experiment using a wild-type Lewis rat was performed to exclude any signal generation due to a photoelectric effect. A similar experimental setup was used to acquire differential recordings triggered by the same optical stimulation.

Statistical Analysis. All results were reported as mean \pm standard deviation unless otherwise noted. One-way analyses of variance (ANOVA) were performed as appropriate with a significance level set to $p<0.05$

\section{ASSOCIATED CONTENT}

\section{(3) Supporting Information}

The Supporting Information is available free of charge at https://pubs.acs.org/doi/10.1021/acsabm.0c00401.

Shear recovery curves, cyclic voltammetry curves, AFM nanoindentation curves, SEM images of collagen-I attachment, neural cell attachment images, signal spatial distribution, and noise levels of $\mu$-ECoG recordings (PDF)

\section{AUTHOR INFORMATION}

\section{Corresponding Author}

Stéphanie P. Lacour - Bertarelli Foundation Chair in Neuroprosthetic Technology, Laboratory for Soft Bioelectronic Interfaces, Institute of Microengineering, Institute of Bioengineering, Center for Neuroprosthetics, Ecole Polytechnique Féderale de Lausanne (EPFL), Geneva 1202, Switzerland; ○ orcid.org/0000-0001-9075-4022;

Email: stephanie.lacour@epfl.ch

\section{Authors}

Michael Shur - Bertarelli Foundation Chair in Neuroprosthetic Technology, Laboratory for Soft Bioelectronic Interfaces, Institute of Microengineering, Institute of Bioengineering, Center for Neuroprosthetics, Ecole Polytechnique Féderale de Lausanne (EPFL), Geneva 1202, Switzerland; (1) orcid.org/0000-00032545-9777

Florian Fallegger - Bertarelli Foundation Chair in Neuroprosthetic Technology, Laboratory for Soft Bioelectronic Interfaces, Institute of Microengineering, Institute of Bioengineering, Center for Neuroprosthetics, Ecole Polytechnique Féderale de Lausanne (EPFL), Geneva 1202, Switzerland; (1) orcid.org/0000-0003-0626-5264

Elvira Pirondini - Department of Radiology and Medical Informatics, University of Geneva, Geneva 1211, Switzerland; Defitech Center for Interventional Neurotherapies (NeuroRestore), Department of Neurosurgery, University Hospital of Lausanne (CHUV) and University of Lausanne (UNIL), Lausanne 1015, Switzerland

Adrien Roux - Tissue Engineering Laboratory, HEPIA - HESSO University of Applied Sciences and Arts Western Switzerland, Geneva 1202, Switzerland; Swiss Center for Applied Human Toxicology (SCAHT), Basel 4055, Switzerland

Arnaud Bichat - Bertarelli Foundation Chair in Neuroprosthetic Technology, Laboratory for Soft Bioelectronic Interfaces, Institute of Microengineering, Institute of Bioengineering, Center for Neuroprosthetics and Center for Neuroprosthetics and Brain Mind Institute, School of Life Sciences, Ecole Polytechnique Féderale de Lausanne (EPFL), Geneva 1202, Switzerland; Defitech Center for Interventional Neurotherapies (NeuroRestore), Department of Neurosurgery, University Hospital of Lausanne (CHUV) and University of Lausanne (UNIL), Lausanne 1015, Switzerland

Quentin Barraud - Center for Neuroprosthetics and Brain Mind Institute, School of Life Sciences, Ecole Polytechnique Federale de Lausanne (EPFL), Lausanne 1002, Switzerland; Defitech Center for Interventional Neurotherapies

(NeuroRestore), Department of Neurosurgery, University Hospital of Lausanne (CHUV) and University of Lausanne (UNIL), Lausanne 1015, Switzerland

Grégoire Courtine - Center for Neuroprosthetics and Brain Mind Institute, School of Life Sciences, Ecole Polytechnique Fédérale de Lausanne (EPFL), Lausanne 1002, Switzerland; Defitech Center for Interventional Neurotherapies (NeuroRestore), Department of Neurosurgery, University Hospital of Lausanne (CHUV) and University of Lausanne (UNIL), Lausanne 1015, Switzerland

Complete contact information is available at: 
https://pubs.acs.org/10.1021/acsabm.0c00401

\section{Notes}

The authors declare no competing financial interest.

\section{ACKNOWLEDGMENTS}

This work is supported by the Swiss National Science Foundation (grant BSCGI0_157800), the Bertarelli Foundation, and the Wyss Center for Bio and Neuroengineering in Geneva, Switzerland. The authors thank Prof. Jocelyne Bloch for her support of the activities of E.P. The authors also thank Laetitia Nikles for her help with the in vitro study and Elizabeth Canovic and Laetitia Baud for their assistance with the Thy-1 rat colony.

\section{REFERENCES}

(1) Donoghue, J. P. Bridging the Brain to the World: A Perspective on Neural Interface Systems. Neuron 2008, 60, 511-521.

(2) Cutrone, A.; Micera, S. Implantable Neural Interfaces and Wearable Tactile Systems for Bidirectional Neuroprosthetics Systems. Adv. Healthcare Mater. 2019, 8, 1801345.

(3) Ferro, M. D.; Melosh, N. A. Electronic and Ionic Materials for Neurointerfaces. Adv. Funct. Mater. 2018, 28, 1704335.

(4) Kostarelos, K.; Vincent, M.; Hebert, C.; Garrido, J. A. Graphene in the Design and Engineering of Next-Generation Neural Interfaces. Adv. Mater. 2017, 29, 1700909.

(5) Venkatraman, S.; Hendricks, J.; King, Z. A.; Sereno, A. J.; Richardson-Burns, S.; Martin, D.; Carmena, J. M. In Vitro and In Vivo Evaluation of PEDOT Microelectrodes for Neural Stimulation and Recording. IEEE Trans. Neural Syst. Rehabil. Eng. 2011, 19, 307-316.

(6) Ecker, M.; Joshi-Imre, A.; Modi, R.; Frewin, C. L.; GarciaSandoval, A.; Maeng, J.; Gutierrez-Heredia, G.; Pancrazio, J. J.; Voit, W. E. From Softening Polymers to Multi-Material Based Bioelectronic Devices. Multifunct. Mater. 2018, 2, No. 012001.

(7) Fattahi, P.; Yang, G.; Kim, G.; Abidian, M. R. A Review of Organic and Inorganic Biomaterials for Neural Interfaces. Adv. Mater. 2014, 26, 1846-1885.

(8) Szostak, K. M.; Grand, L.; Constandinou, T. G. Neural Interfaces for Intracortical Recording: Requirements, Fabrication Methods, and Characteristics. Front. Neurosci. 2017, 11, 665.

(9) Cogan, S. F.; Ehrlich, J.; Plante, T. D.; Smirnov, A.; Shire, D. B.; Gingerich, M.; Rizzo, J. F. Sputtered Iridium Oxide Films for Neural Stimulation Electrodes. J. Biomed. Mater. Res. B Appl. Biomater. 2009, 89B, 353-361.

(10) Magliulo, M.; Mulla, M. Y.; Singh, M.; Macchia, E.; Tiwari, A.; Torsi, L.; Manoli, K. Printable and Flexible Electronics: From TFTs to Bioelectronic Devices. J. Mater. Chem. C 2015, 3, 12347-12363.

(11) Li, L.; Pan, L.; Ma, Z.; Yan, K.; Cheng, W.; Shi, Y.; Yu, G. All Inkjet-Printed Amperometric Multiplexed Biosensors Based on Nanostructured Conductive Hydrogel Electrodes. Nano Lett. 2018, $18,3322-3327$.

(12) Min, J.; Patel, M.; Koh, W.-G. Incorporation of Conductive Materials into Hydrogels for Tissue Engineering Applications. Polymers 2018, 10, 1078.

(13) Green, R. A.; Baek, S.; Poole-Warren, L. A.; Martens, P. J. Conducting Polymer-Hydrogels for Medical Electrode Applications. Sci. Technol. Adv. Mater. 2010, 11, 014107.

(14) Green, R. A.; Hassarati, R. T.; Goding, J. A.; Baek, S.; Lovell, N. H.; Martens, P. J.; Poole-Warren, L. A. Conductive Hydrogels: Mechanically Robust Hybrids for Use as Biomaterials. Macromol. Biosci. 2012, 12, 494-501.

(15) Ferlauto, L.; D’Angelo, A. N.; Vagni, P.; Airaghi Leccardi, M. J. I.; Mor, F. M.; Cuttaz, E. A.; Heuschkel, M. O.; Stoppini, L.; Ghezzi, D. Development and Characterization of PEDOT:PSS/Alginate Soft Microelectrodes for Application in Neuroprosthetics. Front. Neurosci. 2018, 12, 648
(16) Green, R.; Abidian, M. R. Conducting Polymers for Neural Prosthetic and Neural Interface Applications. Adv. Mater. 2015, 27, $7620-7637$.

(17) Thoniyot, P.; Tan, M. J.; Karim, A. A.; Young, D. J.; Loh, X. J. Nanoparticle-Hydrogel Composites: Concept, Design, and Applications of These Promising Multi-Functional Materials. Adv. Sci. 2015, 2, 1400010 .

(18) Liu, Y.; Liu, J.; Chen, S.; Lei, T.; Kim, Y.; Niu, S.; Wang, H.; Wang, X.; Foudeh, A. M.; Tok, J. B.-H.; Bao, Z. Soft and Elastic Hydrogel-Based Microelectronics for Localized Low-Voltage Neuromodulation. Nat. Biomed. Eng. 2019, 3, 58-68.

(19) Liu, Y.; McGuire, A. F.; Lou, H.-Y.; Li, T. L.; Tok, J. B.-H.; Cui, B.; Bao, Z. Soft Conductive Micropillar Electrode Arrays for Biologically Relevant Electrophysiological Recording. Proc. Natl. Acad. Sci. 2018, 115, 11718.

(20) Feig, V. R.; Tran, H.; Lee, M.; Bao, Z. Mechanically Tunable Conductive Interpenetrating Network Hydrogels That Mimic the Elastic Moduli of Biological Tissue. Nat. Commun. 2018, 9, 2740.

(21) Feig, V. R.; Tran, H.; Lee, M.; Liu, K.; Huang, Z.; Beker, L.; Mackanic, D. G.; Bao, Z. An Electrochemical Gelation Method for Patterning Conductive PEDOT:PSS Hydrogels. Adv. Mater. 2019, 31, 1902869.

(22) Kleber, C.; Bruns, M.; Lienkamp, K.; Rühe, J.; Asplund, M. An Interpenetrating, Microstructurable and Covalently Attached Conducting Polymer Hydrogel for Neural Interfaces. Acta Biomater. 2017, $58,365-375$.

(23) Kleber, C.; Lienkamp, K.; Rühe, J.; Asplund, M. Wafer-Scale Fabrication of Conducting Polymer Hydrogels for Microelectrodes and Flexible Bioelectronics. Adv. Biosyst. 2019, 3, 1900072.

(24) Yang, T.-H. Recent Applications of Polyacrylamide as Biomaterials. Recent Pat. Mater. Sci. 2008, 1, 29-40.

(25) Denisin, A. K.; Pruitt, B. L. Tuning the Range of Polyacrylamide Gel Stiffness for Mechanobiology Applications. ACS Appl. Mater. Interfaces 2016, 8, 21893-21902.

(26) Minev, I. R.; Musienko, P.; Hirsch, A.; Barraud, Q.; Wenger, N.; Moraud, E. M.; Gandar, J.; Capogrosso, M.; Milekovic, T.; Asboth, L.; Torres, R. F.; Vachicouras, N.; Liu, Q.; Pavlova, N.; Duis, S.; Larmagnac, A.; Voros, J.; Micera, S.; Suo, Z.; Courtine, G.; Lacour, S. P. Electronic Dura Mater for Long-Term Multimodal Neural Interfaces. Science 2015, 347, 159-163.

(27) Minev, I. R.; Wenger, N.; Courtine, G.; Lacour, S. P. Research Update: Platinum-Elastomer Mesocomposite as Neural Electrode Coating. APL Mater. 2015, 3, No. 014701.

(28) Raja Ashok, R. P.; Thomas, M. S.; Varughese, S. Multi-Region to Single Region Shear Thinning Transitions in Drying PEDOT:PSS Dispersions: Contributions from Charge Density Fluctuations. Soft Matter 2015, 11, 8441-8451.

(29) Elschner, A.; Kirchmeyer, S.; Lovenich, W.; Merker, U.; Reuter, K. PEDOT: Principles and Applications of an Intrinsically Conductive Polymer; CRC Press: 2010, DOI: 10.1201/b10318.

(30) Kardashian, V.; Vellanki, S. A Method for the Rheological Characterization of Thick-Film Pastes. IEEE Trans. Compon. Hybrids Manuf. Technol. 1979, 2, 232-239.

(31) Alias, R.; Mohd, S. Rheological Behaviors and Their Correlation with Printing Performance of Silver Paste for LTCC Tape. In Rheology; De Vicente, J., Ed.; InTech, 2012, DOI: 10.5772/ 35004.

(32) Lacour, S. P.; Wagner, S.; Huang, Z.; Suo, Z. Stretchable Gold Conductors on Elastomeric Substrates. Appl. Phys. Lett. 2003, 82, 2404-2406.

(33) Schiavone, G.; Fallegger, F.; Kang, X.; Barra, B.; Vachicouras, N.; Roussinova, E.; Furfaro, I.; Jiguet, S.; Seáñez, I.; Borgognon, S.; Rowald, A.; Li, Q.; Qin, C.; Bézard, E.; Bloch, J.; Courtine, G.; Capogrosso, M.; Lacour, S. P. Soft, Implantable Bioelectronic Interfaces for Translational Research. Adv. Mater. 2020, 32, 1906512.

(34) Briones, N.; Choong, V.-E.; Johnson, W. Method for Adhesion of Polymers to Metal-Coated Substrates. WO 2002/025282 A2, March 28, 2002. 
(35) Robinson, D. A. The Electrical Properties of Metal Microelectrodes. Proc. IEEE 1968, 56, 1065-1071.

(36) Cogan, S. F. Neural Stimulation and Recording Electrodes. Annu. Rev. Biomed. Eng. 2008, 10, 275-309.

(37) Flores-Merino, M. V.; Chirasatitsin, S.; LoPresti, C.; Reilly, G. C.; Battaglia, G.; Engler, A. J. Nanoscopic Mechanical Anisotropy in Hydrogel Surfaces. Soft Matter 2010, 6, 4466.

(38) Guvendiren, M.; Yang, S.; Burdick, J. A. Swelling-Induced Surface Patterns in Hydrogels with Gradient Crosslinking Density. Adv. Funct. Mater. 2009, 19, 3038-3045.

(39) Lee, Y.-Y.; Kang, H.-Y.; Gwon, S. H.; Choi, G. M.; Lim, S.-M.; Sun, J.-Y.; Joo, Y.-C. A Strain-Insensitive Stretchable Electronic Conductor: PEDOT:PSS/Acrylamide Organogels. Adv. Mater. 2016, $28,1636-1643$.

(40) Sumizawa, T.; Igisu, H. Suppression of Acrylamide Toxicity by Carboxyfullerene in Human Neuroblastoma Cells in Vitro. Arch. Toxicol. 2009, 83, 817-824.

(41) Mehri, S.; Abnous, K.; Mousavi, S. H.; Shariaty, V. M.; Hosseinzadeh, H. Neuroprotective Effect of Crocin on AcrylamideInduced Cytotoxicity in PC12 Cells. Cell. Mol. Neurobiol. 2012, 32, 227-235.

(42) Roach, P.; Parker, T.; Gadegaard, N.; Alexander, M. R. Surface Strategies for Control of Neuronal Cell Adhesion: A Review. Surf. Sci. Rep. 2010, 65, 145-173.

(43) Gunn, J. W.; Turner, S. D.; Mann, B. K. Adhesive and Mechanical Properties of Hydrogels Influence Neurite Extension. J. Biomed. Mater. Res. 2005, 72A, 91-97.

(44) Simitzi, C.; Ranella, A.; Stratakis, E. Controlling the Morphology and Outgrowth of Nerve and Neuroglial Cells: The Effect of Surface Topography. Acta Biomater. 2017, 51, 21-52.

(45) Chapman, C. A. R.; Wang, L.; Chen, H.; Garrison, J.; Lein, P. J.; Seker, E. Nanoporous Gold Biointerfaces: Modifying Nanostructure to Control Neural Cell Coverage and Enhance Electrophysiological Recording Performance. Adv. Funct. Mater. 2017, 27, 1604631 .

(46) Tomita, H.; Sugano, E.; Fukazawa, Y.; Isago, H.; Sugiyama, Y.; Hiroi, T.; Ishizuka, T.; Mushiake, H.; Kato, M.; Hirabayashi, M.; Shigemoto, R.; Yawo, H.; Tamai, M. Visual Properties of Transgenic Rats Harboring the Channelrhodopsin-2 Gene Regulated by the Thy1.2 Promoter. PLoS ONE 2009, 4, No. e7679.

(47) Kozai, T. D. Y.; Vazquez, A. L. Photoelectric Artefact from Optogenetics and Imaging on Microelectrodes and Bioelectronics: New Challenges and Opportunities. J. Mater. Chem. B 2015, 3, 49654978 .

(48) Liu, Q.; Singh, A.; Lalani, R.; Liu, L. Ultralow Fouling Polyacrylamide on Gold Surfaces via Surface-Initiated Atom Transfer Radical Polymerization. Biomacromolecules 2012, 13, 1086-1092.

(49) Chen, H.; Zhao, C.; Zhang, M.; Chen, Q.; Ma, J.; Zheng, J. Molecular Understanding and Structural-Based Design of Polyacrylamides and Polyacrylates as Antifouling Materials. Langmuir 2016, 32, 3315-3330.

(50) Zhang, H.; Chiao, M. Anti-Fouling Coatings of Poly (Dimethylsiloxane) Devices for Biological and Biomedical Applications. J. Med. Biol. Eng. 2015, 35, 143-155.

(51) Hu, R.; Cao, Q.; Sun, Z.; Chen, J.; Zheng, Q.; Xiao, F. A Novel Method of Neural Differentiation of PC12 Cells by Using Opti-MEM as a Basic Induction Medium. Int. J. Mol. Med. 2017, 41, 195-201. 